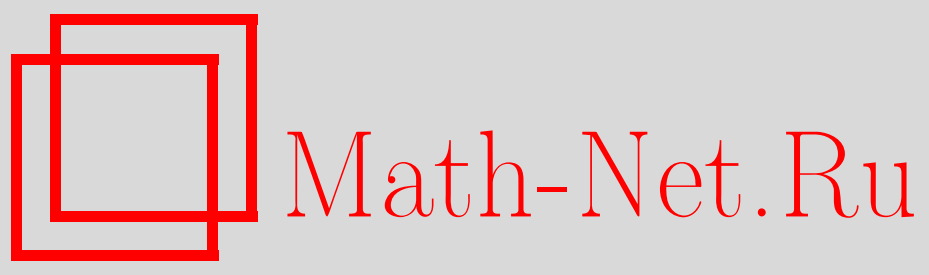

А. М. Вершик, И. М. Гельфанд, С. Г. Гиндикин, А. А. Кириллов, Г. Л. Литвинов, В. Ф. Молчанов, Ю. А. Неретин, В. С. Ретах, Марк Иосифович Граев (к 85-летию со дня рождения), УМН, 2008, том 63, выпуск 1, 169-182

DOI: https://doi.org/10.4213/rm9124

Использование Общероссийского математического портала Math-Net.Ru подразумевает, что вы прочитали и согласны с пользовательским соглашением http://www.mathnet.ru/rus/agreement

Параметры загрузки:

IP : 54.224 .60 .19

26 апреля 2023 г., 15:04:57

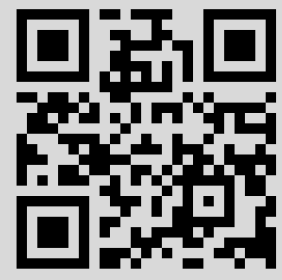




\title{
МАТЕМАТИЧЕСКАЯ ЖКИЗНЬ
}

\author{
Марк Иосифович Граев \\ (к восьмидесятипятилетию со дня рождения)
}

21 ноября 2007 г. исполнилось 85 лет замечательному математику Марку Иосифовичу Граеву. На протяжении многих лет Марк Иосифович является одним из самых авторитетных и признанных специалистов в области теории представлений и ее приложений и основным соавтором одного из создателей современной теории представлений - И. М. Гельфанда.

Отец М.И. родился и вырос в бедной многодетной семье в г. Слуцке, окончил перед Первой мировой войной гимназию с золотой медалью и поступил на математический факультет Петроградского университета. Однако, из-за известных событий тех лет, он вынужден был прервать учебу. До середины 30-х годов отец был политработником в Красной армии, а после увольнения из армии - преподавателем. Военное учреждение, где работал отец (оно помещалось на месте теперешнего Университета дружбы народов),

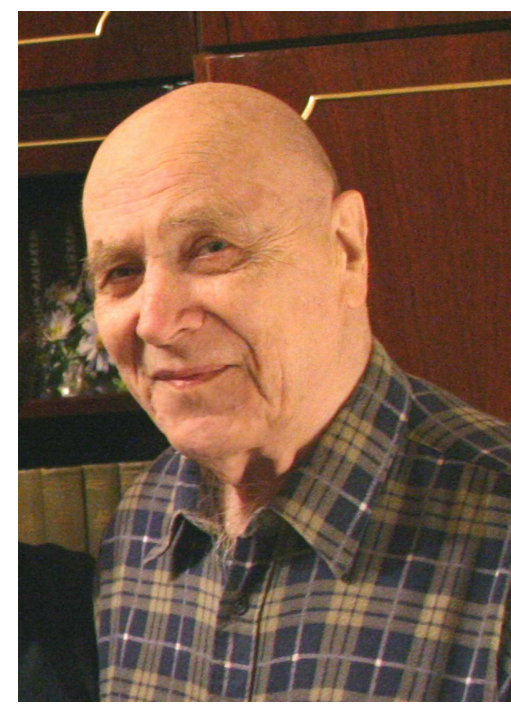
шефствовало над МХАТом, благодаря чему юный М.И. помногу раз пересмотрел почти все спектакли этого театра. Мать М. И. до начала войны заведовала школьной библиотекой. Отец сумел привить М.И. представление о математике как о могущественной науке, дающей ответы на все жизненные вопросы.

В 1930 г. М.И. поступил сразу во второй класс школы. Увлечению и занятиям математикой способствовала молодая учительница математики в младших классах, сумевшая распознать талант своего ученика и внушить ему уверенность в своих силах. С 1937 г. М.И. посещает школьный кружок в МГУ и до сих пор вспоминает тот священный трепет, с которым он переступал порог здания на Моховой. В 1939 г. М. И. получает первую премию на математической олимпиаде в МГУ и вместе с грамотой победителя кипу книг, среди которых книжка П. С. Александрова по теории групп с его автографом, математические труды Ньютона и т. д.

В 1939 г. М. И. оканчивает с отличием школу и поступает на мехмат МГУ. Во время войны М.И. не призывался в армию по состоянию здоровья. В начале войны он вместе с семьей эвакуируется в г. Свердловск, где продолжает учебу в Свердловском государственном университете. Спустя год, после переезда туда из Алма-Аты Московского университета, он возобновляет учебу в МГУ. Там М.И. сближается с А. Г. Курошем и становится участником его семинара, на котором изучалась книга Понтрягина "Непрерывные группы". В 1944 г. М. И. оканчивает с отличием мехмат 
и поступает в аспирантуру к А.Г. Курошу. Первые работы М. И. были посвящены теории абстрактных групп и теории структур. Вскоре под влиянием книги Понтрягина и известной статьи А. А. Маркова по теории свободных топологических групп М. И. начинает заниматься этим предметом, по-новому излагая, упрощая и продолжая работу А.А. Маркова. Этому была посвящена его кандидатская диссертация ("Свободные топологические группы") и статьи [4]-[8]; эта работа была удостоена премии "Молодому математику" Московского математического общества за 1948 г. В дальнейшем исследования М. И. по этой теме были подхвачены в разных странах (школа Неймана в Австралии и др.). Одновременно М. И. преподает на кафедре математического анализа, возглавляемой А. Я. Хинчиным. Поддерживавшие его тогда А.Г. Курош, А.Я. Хинчин и В.В. Степанов предполагали оставить М.И. в МГУ, и с этой целью защита кандидатской диссертации была организована еще в апреле 1947 г., т. е. за полгода до окончания аспирантуры. Но остаться в МГУ не удалось, и М. И. был направлен по распределению в НИИ Академии артиллерийских наук, где работал в должности старшего научного сотрудника до 1951 г. В 1951 г. он перешел на преподавательскую работу в Московский институт стали, где работал до 1958 г.

В 1949 г. М.И. начал ходить на семинар И. М. Гельфанда. И. М. сразу приметил М.И. и дал ему почитать рукопись своей книги с М.А. Наймарком по теории представлений, которая тогда только готовилась к печати. С этого времени началась совместная работа И. М. и М. И. Это плодотворное сотрудничество, продолжавшееся почти 50 лет и давшее математике столько замечательных результатов, представляет собой нечастый феномен в истории науки. М. И. любит вспоминать о первых годах этой совместной работы. Обычно в те годы И. М. звонил ему и приглашал приехать к нему домой на Хавско-Шаболовскую (И. М. жил там в одном доме со своим учеником М. Л. Цетлиным) или на снимаемую где-нибудь под Москвой дачу, в зависимости от времени года. Позже они часто занимались в стенах Института прикладной математики, куда М.И. перешел на работу в 1958 г. по приглашению И. М. Там в отделе Гельфанда он работал с 1958 по 1991 г.; в 1991 г. отдел был переведен в НИИ системных исследований, где М.И. работает в настоящее время. В 1959 г. М. И. защищает докторскую диссертацию "Аналитические функции многих комплексных переменных и представления вещественных простых групп Ли".

Во время занятий с И. М., вспоминает М. И., они не обязательно сидели вдвоем за столом и писали или обсуждали предмет. Обычно у И. М. находилось в течение дня с десяток других дел, ему постоянно звонили, приходили другие люди, начинался разговор с ними совсем на другую тему. Так что занятия шли как бы урывками. Но, как ни странно, в конце дня оказывалось, что был сделан еще один важный шаг, что-то еще стало более понятным. Бывали периоды напряженных занятий. Тяжким трудом для всех была подготовка пятого и шестого выпусков "Обобщенных функций”, которые позже стали настольной книгой нескольких поколений математиков. Однажды, задержавшись допоздна на даче у И. М., М. И. со вторым соавтором по пятому выпуску [35] Н. Я. Виленкиным бежали 6 км до станции, боясь опоздать к последнему поезду, отходившему в 1 час ночи. Когда рукопись пятого выпуска, наконец, была передана в редакцию, авторам казалось, что все уже позади, и их охватило чувство сказочного облегчения. Оказалось, однако, что на этом еще не все закончилось. И. М. подобрал очень строгого редактора - Феликса Широкова. Часто Феликс просиживал у М.И. дома до поздней ночи, придираясь к каждой букве, а потом вызывал такси. Впрочем, иногда использовалась курьерская связь - курьером служила его жена Светлана Аллилуева. Трудной была работа и над шестым выпуском [48], который писался вместе с И.И. Пятецким-Шапиро, еще и потому, что одна из его тем представления групп матриц над неархимедовыми полями - была тогда новой для авторов.

Совместные работы И. М. и М. И. относятся к нескольким разделам математики теории представлений групп, интегральной геометрии (частично с З.Я. Шапиро, 
С.Г. Гиндикиным и др.), представлениям функциональных групп (совместно с А. М. Вершиком), гипергеометрическим функциям (совместно с В. С. Ретахом) и др. K этой работе привлекались молодые люди, темы расширялись, множились, рассказывались на знаменитом семинаре Гельфанда, и история всей этой деятельности, несомненно, будет в дальнейшем предметом тщательного изучения.

М. И. - соавтор трех книг: [35] (в соавторстве с И. М. Гельфандом Н. Я. Виленкиным), [48] (в соавторстве с И. М. Гельфандом и И. И. Пятецким-Шапиро) и [151] (в соавторстве с И. М. Гельфандом и С.Г. Гиндикиным). Эти книги переведены на английский (вторая - дважды), а первая еще и на французский язык. Коллектив авторов последней книги в 1998 г. получил Государственную премию за цикл работ по интегральной геометрии.

М. И. много времени посвятил преподавательской работе и руководству аспирантами; с 1951 по 1958 г. он - доцент в Московском институте стали, и, по совместительству, с 1952 по 1956 г. - доцент МГУ по кафедре высшей алгебры; с 1967 по 1969 г. он работает по совместительству профессором в Коломенском педагогическом институте, а с 1970 по 1980 г. - во Всесоюзном заочном педагогическом институте.

Перечислим бегло основные циклы научных работ М. И. Уже упоминалось, что первый цикл работ М. И., был посвящен свободным топологическим группам. На эту тему после работы А.А. Маркова (1944) осталось много открытых вопросов; часть из них М.И. удалось решить благодаря сделанным им значительным упрощениям и прояснениям теории Маркова. Заметим, что в самое последнее время опять возник интерес к этой теме в связи с проблемами универсальности.

Начальный круг вопросов, к которому И. М. Гельфанд привлек М.И., включал формулу Планшереля для унитарных представлений группы $S L(n, \mathbb{C})$ и теорию неприводимых унитарных представлений вещественных простых групп Ли. Основываясь на свойствах степени квадратичной формы, рассматриваемой как обобщенная функция, они получили новый простой вывод формулы Планшереля. К этому популярному в теории представлений вопросу авторы вернулись позднее в работе [55], сведя вывод формулы Планшереля к одной из задач интегральной геометрии. В работе [12] ими получено описание основных невырожденных серий унитарных представлений группы $S L(n, \mathbb{R})$. После работы Баргмана об унитарных представлениях группы $S L(2, \mathbb{R})$ эта работа явилась первым исследованием по общей теории унитарных представлений вещественных простых групп Ли. Докторская диссертация М. И. была посвящена описанию неприводимых унитарных представлений групп $S U(p, q)$ [22]. В частности, были описаны дискретные серии представлений, реализуемые в пространствах аналитических функций многих переменных, и установлено существование дискретных серий, не обладающих такой реализацией (странные серии). Подробное описание странной серии для случая $S U(2,1)$ приведено в [19].

С конца 50-х И.М. и М.И. начали разрабатывать новый подход к теории представлений - интегральную геометрию в смысле Гельфанда, ставшую одним из самых замечательных открытий гельфандовской теории представлений. Они предложили так называемый метод орисфер, основная идея которого - в сведении задач гармонического анализа на однородных пространствах и задачи обращения преобразования типа Радона к интегрированию по орисферам. Было показано, что в нескольких важных случаях (комплексные полупростые группы Ли, римановы симметрические пространства) формула Планшереля эквивалентна формулам обращения орисферического преобразования [34]. Методом орисфер был решен ряд конкретных задач, в том числе задача о разложении тензорного произведения двух неприводимых представлений основной серии группы $S L(n, \mathbb{C})$ [23]. Ссылки на работу [23] встречаются и в недавних работах специалистов. Все дальнейшее развитие интегральной геометрии в совместных работах И. М. и М.И. связано как с различными применениями к гармоническому анализу на однородных пространствах, так и с рассмотрением 
более общих интегральных преобразований (уже не связанных с группами). Важную роль во многих из этих исследований играют оператор $\kappa$ и понятие допустимых комплексов. Оператор "каппа", введенный в работе [49] (совместной с И. М. и З.Я. Шапиро), относит функциям $\varphi$ на многообразии $k$-мерных плоскостей в $\mathbb{C}^{n}$ дифференциальную форму $\kappa \varphi$ степени $k$ на $\mathbb{C}^{n}$, обладающую следующим свойством: если функция $\varphi$ получена интегрированием функции $f$ на $\mathbb{C}^{n}$ по $k$-мерным плоскостям, то дифференциальная форма $\kappa \varphi$ замкнута на подмногообразиях $M_{x} k$-мерных плоскостей, проходящих через произвольную точку $x$, и

$$
\int_{\gamma_{x}} \kappa \varphi=c_{\gamma_{x}} f(x)
$$

где $\gamma_{x} \subset M_{x}$ - произвольный $k$-мерный цикл. Таким образом, функция $f$ восстанавливается по форме $\kappa \varphi$. Этот оператор позволяет восстановить функцию $f$ на $\mathbb{C}^{n}$ через ее интегралы по некоторым $k$-мерным подмногообразиям $k$-мерных плоскостей (допустимые комплексы). Для случая $k=1$ И. М. и М. И. получили полное геометрическое описание допустимых комплексов [57]. С помощью оператора "каппа" они получили также новый вывод формулы Планшереля для группы $S L(n, \mathbb{C})$. В совместных работах И. М. Гельфанда, С. Г. Гиндикина и М. И. Граева сформулирован и решен ряд задач интегральной геометрии в терминах двойных расслоений [84]. К интегральной геометрии относится более поздняя работа М. И. - статья [165], в которой введено и исследовано интегральное преобразование, относящее функциям $f(x, y)$ на $X \times Y$, где $X$ и $Y$ - произвольные фиксированные локально компактные абелевы группы, функцию $\varphi(a, b)$ на $A \times Y$, где $A$ - группа гомоморфизмов $X \rightarrow Y: \varphi(a, b)=$ $\int_{X} f(x, a x+b) d x$. Это преобразование, являясь обобщением преобразования Радона на плоскости, включает как частный случай классические преобразования Радона в $\mathbb{R}^{n}$ и их многочисленные обобщения на матрицы, любые поля и т. д.

Цикл совместных работ И. М. и М. И. посвящен изучению представлений простых алгебраических групп над конечными и неархимедовыми полями и описанию некоторых представлений классических простых групп и алгебр Ли в базисе ГельфандаЦетлина. В работе [37] они исследовали неприводимые представления простых алгебраических групп над конечным полем; установлено, что каждое из них содержится хотя бы в одном представлении, индуцированном одномерным представлением максимальной нильпотентной подгруппы, и притом с кратностью, не большей единицы; однако этим свойством обладают не все простые группы. В работах [40], [41], [48] построена теория неприводимых унитарных представлений группы $S L(2, K)$, где $K-$ произвольное локально компактное поле: описание представлений, формулы для следов, теорема Планшереля. В частности, получены интегральные формулы для следов непрерывной и дискретной серий, в том числе и в классическом случае. Попутно введены и изучены некоторые специальные функции над неархимедовым полем (с участием А.А. Кириллова). В работе [46] И. М. и М.И получили формулы для операторов конечномерных неприводимых представлений группы $S L(n, \mathbb{C})$ в базисе Гельфанда-Цетлина, точнее, были проинтегрированы формулы для операторов представления ее алгебры Ли. Введены схемы более общего вида, чем схемы ГельфандаЦетлина, в которых неравенства $m_{i j} \geqslant m_{i-1, j} \geqslant m_{i, j+1}$ заменены другими неравенствами. На таких схемах в [46], [51] получены формулы для операторов дискретных серий группы $S L(2, \mathbb{R})$ и формулы для операторов дискретных серий алгебры Ли $\mathfrak{s u}(p, q)$. В работе [171] введены аналоги схем Гельфанда-Цетлина, в которых целые числа $m_{i j}$ заменены унитарными характерами мультипликативной группы $\mathbb{C}^{*}$. В гильбертовом пространстве функций на многообразии таких схем реализована модель основной серии неприводимых унитарных представлений группы $G L(n, \mathbb{C})$, аналогичная модели конечномерных неприводимых представлений этой группы на схемах Гельфанда-Цетлина. 
В начале 70-х М.И. участвует в новом цикле работ с И.М. и А. М. Вершиком по теории представлений бесконечномерных групп - так называемых групп токов и групп диффеоморфизмов. В первой работе [65] был построен "интеграл" представлений группы, т.е. неприводимое представление группы $S L(2, R(X)$ ) (здесь $R(X)$ кольцо функций на многообразии $X$ ), инвариантное относительно преобразований базы, сохраняющих меру. Решающую роль в конструкции играет особое представление $S L(2, \mathbb{R})$, приклеенное к единице, и нетривиальный 1-коцикл в нем. В [66] были описаны группы ранга 1 и их представления, в которых первые когомологии нетривиальны. Эта проблематика в дальнейшем получила развитие в работах этих авторов в нескольких направлениях, в частности, были построены представления группы гладких токов с компактной группой коэффициентов [73], [86], представления групп диффеоморфизмов, связанные с пространством конфигураций [75], и др. Работы [90], [100] по коммутативным моделям представлений групп токов были продолжены позже (см. далее).

В 80-90-х годах М.И. совместно с И. М. и другими соавторами исследует общую теорию гипергеометрических функций и ее связь с вопросами геометрии грассманианов и теории дифференциальных уравнений. В [109] получено описание решений общей геометрической системы, связанной с грассманианом $G_{3,6}$, и их ограничений на страты в $G_{3,6}$. Это первый нетривиальный случай после грассманиана $G_{2,4}-$ для $G_{2,4}$ система сводится к уравнению Гаусса. В работе [106] (совместно с И. М. и А. В. Зелевинским) введена общая гипергеометрическая система уравнений, включающая как частный случай системы, связанные с грассманианами, и доказана ее голономность. Заметим, что многие авторы ссылаются на более позднюю публикацию Гельфанда-Капранова-Зелевинского, в которой проводится дальнейшее изучение этой системы, и называют ее GKZ-системой. В цикле работ, совместных с И. М. и другими соавторами [130], [132], [135], [136], [138] исследованы решения конкретных гипергеометрических систем. В работе [144] (совместной с И. М. и В. С. Ретахом) введены и исследованы $q$-аналоги общих гипергеометрических функций. В [142] с каждой решеткой в $\mathbb{C}^{N}$ связана дифференциально-разностная система уравнений (GG-система), сводящаяся в случае целочисленной решетки к общей системе дифференциальных уравнений, и исследованы ее решения в форме рядов гипергеометрического типа и интегралов. В статье [166] (совместной с И. М. и В. С. Ретахом) введены общие гипергеометрические функции над произвольным локально компактным полем и описаны их решения в форме рядов гипергеометрического типа.

В последние годы (2004-2007) М. И. совместно с А. М. Вершиком продолжил занятия теорией представлений групп токов [170]-[172]. Оказалось, что на результаты работ [65], [100] можно посмотреть с новой точки зрения, а именно строить сначала представления максимальных параболических подгрупп токов, диагонализуя их относительно максимальной унипотентной подгруппы и продолжая затем представление на всю группу. При этом пространство представления реализуется не как фоковское пространство, как это было в старых работах, начиная с работы Х. Араки, а как пространство квадратично интегрируемых функций по замечательной мере бесконечномерной мере Лебега, изученной в последние годы. Попутно здесь были получены новые факты о представлениях параболических подгрупп в простых группах Ли ранга 1.

Исключительная научная добросовестность, преданность науке, поразительная работоспособность и трудолюбие, широта образования, личная скромность и доброта вот качества, отличающие Марка Иосифовича Граева и высоко оцененные его друзьями и коллегами. Пожелаем ему дальнейших научных успехов, здоровья и долголетия.

А.М. Вершик, И.М. Гельфанд, С.Г. Гиндикин, А.А. Кириллов, Г. Л. Литвинов, В. Ф. Молчанов, Ю.А. Неретин, В. С. Ретах 


\section{Список научных трудов М. И. Граева}

[51] “Неприводимые представления алгебры Ли группы $U(p, q)$ ”, Физика высоких энергий и теория элементарных частии, Наукова думка, Киев, 1967, 216-226 (совм. с И. М. Гельфандом).

[52] "Интегральная геометрия на $k$-мерных плоскостях", Функи. анализ и его прил., 1:1 (1967), 15-31 (совм. с И. М. Гельфандом, З. Я. Шапиро); англ. пер.: "Integral geometry on $k$-dimensional planes", Funct. Anal. Appl., 1:1 (1967), 14-27 (with I. M. Gel'fand, Z. Ya. Shapiro).

[53] "Представления группы кватернионов над несвязным локально компактным непрерывным полем”, Докл. АН СССР, 177:1 (1967), 17-20 (совм. с И. М. Гельфандом); англ. пер.: "Representations of the group of quaternions over a disconnected locally compact continuous field", Soviet Math. Dokl., 8 (1967), 1346-1349 (with I. M. Gel'fand).

[54] "Представления групп кватернионов над локально компактными и функциональными полями", Функи. анализ и его прил., 2:1 (1968), 20-35 (совм. с И. М. Гельфандом); англ. пер.: "Representations of quaternion groups over locally compact and functional fields", Funct. Anal. Appl., 2:1 (1968), 19-33 (with I. M. Gel'fand).

[55] "Комплексы $k$-мерных плоскостей в пространстве $C^{n}$ и формула Планшереля для группы $G L(n, C)$ ", Докл. AH CCCP, 179:3 (1968), 522-525 (совм. с И. М. Гельфандом); англ. пер.: "Complexes of $k$-dimensional planes in the space $C^{n}$ and Plancherel's formula for the group $G L(n, C)$ ", Soviet Math. Dokl., 9:1 (1968), 394-398 (with I. M. Gel'fand).

[56] “Теория представлений групп”, Труды Международного конгресса математиков (Москва, 1966), Мир, М., 1968, 373-379 (совм. с А. А. Кирилловым); англ. пер.: "Theory of group representations", Amer. Math. Soc. Transl. Ser. 2, 70 (1968), 50-56 (with A. A. Kirillov).

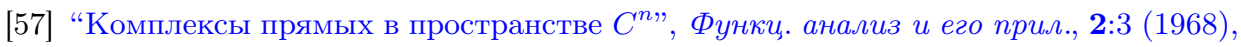
39-52 (совм. с И. М. Гельфандом); англ. пер.: "Complexes of straight lines in the space $C^{n ", ~ F u n c t . ~ A n a l . ~ A p p l ., ~ 2: 3 ~(1968), ~ 219-229 ~(w i t h ~ I . ~ M . ~ G e l ' f a n d) . ~}$

[58] “Дифференциальные формы и интегральная геометрия", Функи. анализ и его прил., 3:2 (1969), 24-40 (совм. с И. М. Гельфандом, З. Я. Шапиро); англ. пер.: "Differential forms and integral geometry", Funct. Anal. Appl., 3:2 (1969), 101-114 (with I. M. Gel'fand, Z. Ya. Shapiro).

[59] "Интегральная геометрия в проективном пространстве", Функи. анализ и его прил., 4:1 (1970), 14-32 (совм. с И. М. Гельфандом, З.Я. Шапиро); англ. пер.: "Integral geometry in projective space", Funct. Anal. Appl., 4:1 (1970), 12-28 (with I. M. Gel'fand, Z. Ya. Shapiro).

[60] "Задача интегральной геометрии, связанная с парой грассмановых многообразий”, Докл. АН СССР, 193:2 (1970), 259-262 (совм. с И. М. Гельфандом, З. Я. Шапиро); англ. пер.: "A problem of integral geometry connected with a pair of Grassmann manifolds", Soviet Math. Dokl., 11 (1970), 892-896 (with I. M. Gel'fand, Z. Ya. Shapiro).

[61] "Классификация линейных представлений группы $S L(2, \mathbb{C})$ ", Докл. AH CCCP, 194:5 (1970), 1002-1005 (совм. с И. М. Гельфандом, В. А. Пономаревым); англ. пер.: "The classification of the linear representations of the group $S L(2, \mathbb{C})$ ", Soviet Math. Dokl., 11 (1970), 1319-1323 (with I. M. Gel'fand, V. A. Ponomarev).

[62] "Однородные обобщенные функции в векторном пространстве над локальным неархимедовым полем, связанные с квадратичной формой”, Функи. анализ и его

Начало списка опубликовано в “Математика в СССР за сорок лет. 1917-1957”, т. 2, Физматгиз, М., 1959, “Математика в СССР 1958-1967”, т. 2, Наука, М., 1970 
прил., 6:3 (1972), 70-71 (совм. с Р. И. Прохоровой); англ. пер.: "Homogeneous generalized functions in a vector space over a local non-Archimedean field which are related to a quadratic form", Funct. Anal. Appl., 6:3 (1972), 233-234 (with R. I. Prokhorova).

[63] "Преобразование Радона в пространстве $P^{n}(A)$, где $A$ - конечное коммутативное кольцо с единицей", Докл. АН CCCP, 207:2 (1972), 270-273 (совм. с Н. В. Павленко); англ. пер.: "The Radon transformation in the space $P^{n}(A)$ where $A$ is a finite commutative ring with identity", Soviet Math. Dokl., 13 (1972), 1493-1497 (with N. V. Pavlenko).

[64] Операторы Фуръе-Вейля на основном аффинном пространстве группы Шевалле, Препринт ИПМ АН СССР № 79, 1973 (совм. с И. М. Гельфандом).

[65] "Представления группы $S L(2, \mathbf{R})$, где $\mathbf{R}$ - кольцо функций”, УMH, 28:5 (1973), 83-128 (совм. с А. М. Вершиком, И. М. Гельфандом); англ. пер.: "Representations of the group $S L(2, \mathbf{R})$, where $\mathbf{R}$ is a ring of functions which are related to a quadratic form", Russ. Math. Surv., 28:5 (1973), 87-132 (with A. M. Vershik, I. M. Gel'fand).

[66] "Неприводимые представления группы $G^{X}$ и когомологии", Функи. анализ и его прил., 8:2 (1974), 67-69 (совм. с А. М. Вершиком, И. М. Гельфандом); англ. пер.: "Irreducible representations of the group $G^{X}$ and cohomologies", Funct. Anal. Appl., 8:2 (1974), 151-153 (with A. M. Vershik, I. M. Gel'fand).

[67] "Обобщенная функция $\pi(P)$, где $P$ - квадратичная форма в векторном пространстве над локальным неархимедовым полем", Гармонический анализ на группах. Сб. научн. трудов, № 39, МГЗПИ, М., 1974 (совм. с Р. И. Прохоровой).

[68] "Квадратные корни из квазирегулярного представления группы $S L(2, K)$ ", Функи. анализ и его прил., 9:2 (1975), 64-66 (совм. с И. М. Гельфандом); англ. пер.: "Square roots of the quasi-regular representation of the group $S L(2, K)$ ", Funct. Anal. Appl., 9:2 (1975), 146-148 (with I. M. Gel'fand).

[69] Представления группы диффеоморфизмов, связанные с бесконечными конфигурациями, Препринт ИПМ АН СССР № 46, 1975 (совм. с И. М. Гельфандом, А. М. Вершиком).

[70] "Представления группы диффеоморфизмов", УМН, 30:6 (1975), 3-50 (совм. с А. М. Вершиком, И. М. Гельфандом); англ. пер.: "Representations of the group of diffeomorphisms", Russ. Math. Surv., 30:6 (1975), 1-50 (with A. M. Vershik, I. M. Gel'fand).

[71] Представления группы гладких отображений многообразия X в компактную әрупnу Ли, Препринт ИПМ АН СССР № 55, 1976 (совм. с И. М. Гельфандом, А. М. Вершиком).

[72] "Representations of the group of smooth mappings of a manifold $X$ into a compact Lie group", Compos. Math., 35:3 (1977), 299-334 (with I. M. Gelfand, A. M. Vershik).

[73] "Представления группы гладких отображений многообразия $X$ в компактную группу Ли", Докл. АН СССР, 232:4 (1977), 745-748 (совм. с А. М. Вершиком, И. М. Гельфандом); англ. пер.: "Representations of the group of smooth mappings from a manifold X into a compact Lie group", Soviet Math. Dokl., 18 (1977), 118-121 (with A. M. Vershik, I. M. Gel'fand).

[74] Замечания о представлениях группы функиий со значениями в компактной групnе Ли, Препринт ИПМ АН СССР № 17, 1979 (совм. с И. М. Гельфандом, А. М. Вершиком).

[75] Задачи интегральной геометрии, связанные с интегрированием дифференциалъных по прямым в $\mathbb{R}^{3} u \mathbb{C}^{3}$, Препринт ИПМ АН СССР № 24, 1979 (совм. с И. М. Гельфандом, С. Г. Гиндикиным). 
[76] Задачи интегральной геометрии, связанные с интегрированием дифференциальных форм по прямым в трехмерном проективном пространстве, Препринт ИПМ АН СССР № 41, 1979 (совм. с И. М. Гельфандом, С. Г. Гиндикиным).

[77] Двойные расслоения и задачи интегральной геометрии, связанные с интегрированием дифференииальных форм по прямым, Препринт ИПМ АН СССР № 60, 1979 (совм. с И. М. Гельфандом, С. Г. Гиндикиным).

[78] Задачи интегральной геометрии в проективном пространстве, свлзанные с интегрированием дифференциальных форм, Препринт ИПМ АН СССР № 79, 1979 (совм. с И. М. Гельфандом, С. Г. Гиндикиным).

[79] Некоторые вопросы, связанные с интегрированием дифференциалъных форм по плоскостям в $\mathbb{R P}^{n} u \mathbb{C P}^{n}$, Препринт ИПМ АН СССР № 126, 1979 (совм. с И. М. Гельфандом, С. Г. Гиндикиным).

[80] Задачи интегральной геометрии в аффинном пространстве, связанные с интегрированием функиий по плоскостям, Препринт ИПМ АН СССР № 152, 1979 (совм. с И. М. Гельфандом, С. Г. Гиндикиным).

[81] "Задача интегральной геометрии в $\mathbb{R P}^{n}$, связанная с интегрированием дифференциальных форм", Функи. анализ и его прил., 13:4 (1979), 64-66 (совм. с И. М. Гельфандом, С.Г. Гиндикиным); англ. пер.: "A problem of integral geometry in $\mathbb{R P}^{n}$, connected with the integration of differential forms", Funct. Anal. Appl., 13:4 (1979), 288-290 (with I. M. Gel'fand, S. G. Gindikin).

[82] Интегральная геометрия для некоторых одномерных расслоений над проективным пространством, Препринт ИПМ АН СССР № 24, 1980 (совм. с И. М. Гельфандом, С. Г. Гиндикиным).

[83] Интегральная геометрия для одномерных расслоений общего вида над $\mathbb{R P}^{n}$, Препринт ИПМ АН СССР № 60, 1980 (совм. с И. М. Гельфандом, С. Г. Гиндикиным).

[84] "Интегральная геометрия в аффинном и проективном пространствах", Итоги науки и техники, Соврем. проблемы матем., 16, ВИНИТИ СССР, М., 1980, 53-226 (совм. с И. М. Гельфандом, С. Г. Гиндикиным).

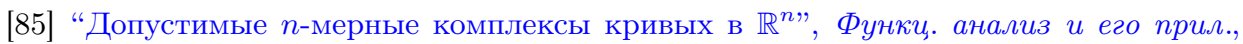
14:4 (1980), 36-44 (совм. с И. М. Гельфандом); англ. пер.: "Admissible $n$-dimensional complexes of curves in $\mathbb{R}^{n}$ ", Funct. Anal. Appl., 14:4 (1980), 274-281 (with I. M. Gel'fand).

[86] "Representations in the group of functions taking values of a compact Lie group", Compos. Math., 42:2 (1981), 217-243 (with I. M. Gel'fand, A. M. Vershik).

[87] Интегральные преобразования, связанные с двумя замечательными комплексами прямых в проективном пространстве, Препринт ИПМ АН СССР № 93, 1982 (совм. с И. М. Гельфандом).

[88] "Нелокальные формулы обращения в задаче интегральной геометрии, связанной с $p$-мерными плоскостями в вещественном проективном пространстве", Функи. анализ и его прил., 16:3 (1982), 49-51 (совм. с И. М. Гельфандом, Р. Рошу); англ. пер.: "Nonlocal inversion formulas in a problem of integral geometry related to $p$-dimensional planes in real projective space", Funct. Anal. Appl., 16:3 (1982), 196-198 (with I. M. Gel'fand, R. Roshu).

[89] “Формула Планшереля для интегрального преобразования, связанного с комплексом прямых, пересекающих алгебраическую кривую в $\mathbb{C}^{3}$ и $\mathbb{C P}^{3}$ ", Докл. АН АрмССР, 75:1 (1982), 9-15 (совм. с Р. Г. Айрапетяном, И. М. Гельфандом, Г. Р. Оганесяном).

[90] Коммутативная модель основного представления группы $\mathrm{SL}(2, \mathbb{R})^{X}$ относительно унипотентной подгруппы, Препринт ИПМ АН СССР № 169, 1982 (совм. с И. М. Гельфандом, А. М. Вершиком). 
[91] “Теорема Планшереля для интегрального преобразования, связанного с комплексом $p$-мерных плоскостей в $\mathbb{C P}^{n} "$, Докл. АН Арм ССР, 268:2 (1982), 265-268 (совм. с Р. Г. Айрапетяном, И. М. Гельфандом, Г. Р. Оганесяном).

[92] "Коммутативная модель представления группы токов $S L(2, \mathbb{R})^{X}$, связанная с унипотентной подгруппой”, Функи. анализ и его прил., 17:2 (1983), 70-72 (совм. с А.М. Вершиком, И. М. Гельфандом); англ. пер.: "A commutative model of representation of the group of flows $S L(2, \mathbb{R})^{X}$ that is connected with a unipotent subgroup", Funct. Anal. Appl., 17:2 (1983), 137-139 (with A. M. Vershik, I. M. Gel'fand).

[93] "Коммутативная модель основного представления группы $G L(2, \mathbb{R})^{X}$ относительно унипотентной подгруппы”, Теоретико-групповые методы в физике, $\mathbf{2}$, Наука, М., 1983, 472-487 (совм. с И. М. Гельфандом, А. М. Вершиком).

[94] "The problem of integral geometry for $p$-dimensional planes in real projective space (the non-local variant)", Operator algebras and group representations, Vol. 1 (Neptun, 1980), Monogr. Stud. Math., 17, Pitman, Boston, MA, 1984, 192-207 (with I. M. Gelfand, R. Roshu).

[95] The problem of integral geometry and intertwining operators for a pair of real Grassmannian manifolds, Preprint 42, INCREST, Romania, 1983 (with I. M. Gelfand, R. Roshu).

[96] "The problem of integral geometry and intertwining operators for a pair of real Grassmannian manifolds", J. Operator Theory, 12:2 (1984), 359-383 (with I. M. Gelfand, R. Roshu).

[97] "Теорема Планшереля для интегрального преобразования, связанного с комплексом $p$-мерных плоскостей в $\mathbb{C P}^{n}$ и $\mathbb{C}^{n} "$, Изв. АН АрмССР. Матем., 18:4 (1983), 271-282 (совм. с Р. Г. Айрапетяном, И. М. Гельфандом, Г. Р. Оганесяном); англ. пер.: "Plancherel theorem for an integral transformation associated with a complex of $p$-dimensional planes in $\mathbb{C P}^{n}$ and $\mathbb{C}^{n}$ ", Soviet J. Contemp. Math. Anal., 18:4 (1983), 21-32 (with R. G. Airapetyan, I. M. Gel'fand, G. R. Oganesyan).

[98] "Теорема Планшереля для интегрального преобразования, связанного с парой грассмановых многообразий", Изв. АН АрмССР. Матем., 19:6 (1984), 467-483 (совм. с Р.Г. Айрапетяном, И. М. Гельфандом, Г. Р. Оганесяном); англ. пер.: "Plancherel's theorem for an integral transformation connected with a pair of Grassmann manifolds", Soviet. J. Contemp. Math. Anal. Arm. Acad. Sci., 19:6 (1984), 53-69 (with R. G. Ajrapetyan, I. M. Gel'fand, G. R. Oganesyan).

[99] O некоторых семействах неприводимых унитарных представлений группь $U(\infty)$, Препринт ИПМ АН СССР № 51, 1985 (совм. с И. М. Гельфандом).

[100] "Models of representations of current groups", Representations of Lie groups and Lie algebras (Budapest, 1971), Akad. Kiadó, Budapest, 1985, 121-179 (with I. M. Gelfand, A. M. Vershik).

[101] Описание всех формул обращения в задаче интегральной геометрии, связанной с грассманианом $G_{k, n}$, Препринт ИПМ АН СССР № 102, 1986 (совм. с И. М. Гельфандом).

[102] “Теорема двойственности для общих гипергеометрических функций”, Докл. АH СССР, 289:1 (1986), 19-23 (совм. с И. М. Гельфандом).

[103] "Гипергеометрические функции, связанные с грассманианом $G_{3,6}$ ", Докл. AH CCCP, 293:2 (1987), 288-293 (совм. с И.М. Гельфандом); англ. пер.: "Hypergeometric functions associated with the Grassmannian $G_{3,6}$ ", Soviet Math. Dokl., 35:2 (1987), 298-303 (with I. M. Gel'fand).

[104] Общие гипергеометрические функиии на грассманиане $G_{3,6}$, Препринт ИПМ АН СССР № 123, 1987 (совм. с И. М. Гельфандом).

[105] Страты в $G_{3,6}$ и связанные с ними общие гипергеометрические функиии, Препринт ИПМ АН СССР № 127, 1987 (совм. с И. М. Гельфандом). 
[106] "Голономные системы уравнений и ряды гипергеометрического типа", Докл. АН СССР, 295:1 (1987), 14-19 (совм. с И. М. Гельфандом, А. В. Зелевинским); англ. пер.: "Holonomic systems of equations and series of hypergeometric type", Soviet Math. Dokl., 36:1 (1988), 5-10 (with I. M. Gel'fand, A. V. Zelevinskij).

[107] "Формула Планшереля, связанная с допустимым комплексом прямых в $\mathbb{C P}^{3}$ ", Изв. АН АрмССР. Матем., 23:5 (1988), 463-488 (совм. с Р. Г. Айрапетяном, М. М. Граевым, Г. Р. Оганесяном); англ. пер.: "Plancherel formula associated with admissible complex of straight lines in $\mathbb{C P}^{3}$ ", Soviet J. Contemporary Math. Anal., 23:5 (1988), 62-90 (with R. G. Ajrapetyan, M. M. Graev, R. G. Oganesyan).

[108] "Integral geometry and separation of continuous and discrete spectra in regular representaton of $\operatorname{PSL}(2, \mathbb{R})$ ", J. Geom. Phys., 5:2 (1988), 183-190.

[109] "Гипергеометрические функции, связанные с грассманианом $G_{3,6}$ ", Матем. сб., 180:1 (1989), 3-38 (совм. с И. М. Гельфандом); англ. пер.: "Hypergeometric functions associated with the Grassmannian $G_{3,6}$ ", Math. USSR-Sb., 66:1 (1990), 1-40 (with I. M. Gel'fand).

[110] Интеграл нормальной кривизны и формула Планшереля в 3-мерной комплексной интегралъной геометрии, Препринт ИПМ АН СССР № 149, 1989 (совм. с Р. Г. Айрапетяном, М. М. Граевым, Г. Р. Оганесяном).

[111] Г-ряды и общие гипергеометрические функиии на многообразии $k \times n$-матрич, preprintinfo Препринт ИПМ № 64, 1990 (совм. с И. М. Гельфандом, В. С. Ретахом).

[112] Гипергеометрические функиии на стратах малых коразмерностей в $G_{k, n}$, Препринт ИПМ № 138, 1990 (совм. с И. М. Гельфандом, В. С. Ретахом).

[113] "Principal representatons of the group U( $\infty)$ ", Adv. Stud. Contemp. Math., 7 (1990), 119-153 (with I. M. Gelfand).

[114] "Функция Крофтона и формулы обращения в вещественной интегральной геометрии”, Функи. анализ и его прил., 25:1 (1991), 1-6 (совм. с И. М. Гельфандом); англ. пер.: "Crofton's function and inversion formulas in real integral geometry", Funct. Anal. Appl., 25:1 (1991), 1-45 (with I. M. Gel'fand).

[115] "Разностные аналоги общих гипергеометрических функций”, XVI школа по теории операторов, Н. Новгород, 1991 (совм. с В. С. Ретахом).

[116] "Формулы приведения для гипергеометрических функций на грассманиане $G_{k, n}$ и описание гипергеометрических функций на стратах малых коразмерностей”, Докл. АН СССР, 318:4 (1991), 793-797 (совм. с И. М. Гельфандом, В. С. Ретахом); англ. пер.: "Reduction formulas for hypergeometric on the Grassmannian $G_{k, n}$, and a description of hypergeometric functions strata of small codimensions", Soviet Math. Dokl., 43:3 (1991), 757-761 (with I. M. Gel'fand, V.S. Retakh).

[117] "Гипергеометрические функции на $k$-й внешней степени пространства $\mathbb{C}^{n}$ и грассманиане $G_{k, n}$ и связь между ними”, Докл. АН СССР, 320:1 (1991), 20-24 (совм. с И. М. Гельфандом, В. С. Ретахом); англ. пер.: "Hypergeometric functions on the $k$ th exterior power of the space $\mathbb{C}^{n}$ and the Grassmannian $G_{k, n}$, and the connection between them", Soviet Math. Dokl., 44:2 (1992), 375-380 (with I. M. Gel'fand, V.S. Retakh).

[118] "Recent developments in the theory of general hypergeometric functions", Special differential equations, Proceedings of the Taniguchi Workshop (Japan, 1991), 69-76 (with I. M. Gelfand, V.S. Retakh).

[119] "Общие гипергеометрические функции”, Функи. анализ и его прил., 26:2 (1992), 69-72; англ. пер.: "General hypergeometric functions", Funct. Anal. Appl., 26:2 (1992), 131-133.

[120] "Обобщенные гипергеометрические функции, связанные с произвольным конечным или локально компактным полем”, Докл. РАН, 323:3 (1992), 394-397 (совм. 
с И.М. Гельфандом, В. С. Ретахом); англ. пер.: "Generalized hypergeometric functions associated with an arbitrary finite or locally compact continuous field", Dokl. Math., 45:2 (1992), 343-347 (with I. M. Gel'fand, V. S. Retakh).

[121] "Общие гипергеометрические системы уравнений и ряды гипергеометрического типа”, УМН, 47:4 (1992), 3-82 (совм. с И. М. Гельфандом, В. С. Ретахом); англ. пер.: "General hypergeometric systems of equations and series of hypergeometric type", Russian Math. Surveys, 47:4 (1992), 1-88 (with I. M. Gel'fand, V. S. Retakh).

[122] "Разностные и $q$-аналоги общих гипергеометрических систем дифференциальных уравнений", Докл. РАН, 325:2 (1992), 215-220 (совм. с И. М. Гельфандом, В.С. Ретахом); англ. пер.: "Difference analogues and $q$-analogues of general hypergeometric systems of differential equations", Dokl. Math., 46:1 (1993), 30-35 (with I. M. Gel'fand, V.S. Retakh).

[123] "GG-функции”, Докл. РАН, 328:6 (1993), 645-648 (совм. с И. М. Гельфандом); англ. пер.: "GG-functions", Dokl. Math., 47:1 (1993), 134-138 (with I. M. Gel'fand).

[124] " $q$-гипергеометрическое уравнение Гаусса и описание его решений в виде рядов и интегралов”, Докл. РАН, 331:2 (1993), 140-143 (совм. с И. М. Гельфандом, В.С. Ретахом); англ. пер.: "The q-hypergeometric equation of Gauss and a description of its series and integral solutions", Dokl. Math., 48:1 (1994), 40-45 (with I. M. Gel'fand, V.S. Retakh).

[125] "Hypergeometric systems of Gelfand-type equations and series in ortogonal polynomials", Lett. Math. Phys., 28:3 (1993), 195-205 (with V. S. Retakh).

[126] "Reduction formulas for hypergeometric functions associated with the Grassmannian $G_{k, n}$ and description of these functions on strata of small codimension in $G_{k, n}$ ", Russian J. Math. Phys., 1:1 (1993), 19-56 (with I. M. Gelfand, V.S. Retakh).

[127] "Особые представления группы $S U(n, 1)$ и проективные унитарные представления группы токов $S U(n, 1)^{X}$,, Докл. РAH, 332:3 (1993), 280-282 (совм. с И. М. Гельфандом); англ. пер.: "Singular representations of the group $S U(n, 1)$ and projective unitary representations of the current group $S U(n, 1)^{X}$ ", Dokl. Math., 48:2 (1994), 291-295 (with I. M. Gel'fand).

[128] “( $R, S)$-гипергеометрические функции одной переменной”, Докл. РАH, 333:5 (1993), 567-570 (совм. с И. М. Гельфандом, В. С. Ретахом); англ. пер.: "( $R, S)$-hypergeometric functions of one variable", Dokl. Math., 48:3 (1994), 591-596 (with I. M. Gel'fand, V.S. Retakh).

[129] "Проективные представления группы токов $S U(1,1)^{X}$ ", Функи. анализ и его прил., 27:4 (1993), 65-68 (совм. с И. М. Гельфандом); англ. пер.: "Projective representations of the current group $S U(1,1)^{X}$ ", Funct. Anal. Appl., 27:4 (1993), 275-277 (with I. M. Gelfand).

[130] "Hypergeometric functions on the external power $\Lambda^{k} \mathbb{C}^{n}$ and on the Grassmannian $G_{k, n}$, their relationships and integral representations", Russian J. Math. Phys., 1:3 (1993), 289-311 (with I. M. Gelfand, V. S. Retakh).

[131] “( $r, s)$-экспоненты”, Докл. РАН, 336:6 (1994), 730-732 (совм. с И. М. Гельфандом, В. С. Ретахом, С. А. Спириным); англ. пер.: "( $r, s)$-exponentials", Dokl. Math., 49:3 (1994), 577-581 (with I. M. Gel'fand, V. S. Retakh, S. A. Spirin).

[132] "Гипергеометрические функции на флаговых многообразиях", Докл. PAH, 338:2 (1994), 154-157 (совм. с И. М. Гельфандом); англ. пер.: "Hypergeometric functions on flag spaces", Dokl. Math., 50:2 (1995), 204-209 (with I. M. Gel'fand).

[133] "Проективные неунитарные представления группы токов", Докл. РАН, 338:3 (1994), 298-301 (совм. с И. М. Гельфандом); англ. пер.: "Projective nonunitary representations of current groups", Dokl. Math., 50:2 (1995), 234-239 (with I. M. Gel'fand). 
[134] "Уравнения и ряды гипергеометрического типа", Функи.. анализ и его прил., 29:1 (1995), 72-76; англ. пер.: "Equations and series of hypergeometric type", Funct. Anal. Appl., 29:1 (1995), 56-59.

[135] "Гипергеометрические функции на пространстве $p$-мерных матриц", Докл. РАН, 346:1 (1996), 7-10 (совм. с И. М. Гельфандом); англ. пер.: "Hypergeometric functions on the space of $p$-dimensional matrices", Dokl. Math., 53:1 (1996), 1-4 (with I. M. Gel'fand).

[136] "Гипергеометрические функции и многогранник Ньютона, связанные с действием тора $\left(\mathbb{C}^{*}\right)$ на $\bigwedge^{k} \mathbb{C}^{n}$ ", Докл. РАН, 348:2 (1996), 155-158 (совм. с И. М. Гельфандом, С. А. Спириным); англ. пер.: "Hypergeometric functions and the Newton polytope associated with the action of the torus $\left(\mathbb{C}^{*}\right)$ on $\bigwedge^{k} \mathbb{C}^{n}$, Dokl. Math., 53:3 (1996), 348-351 (with I. M. Gel'fand, S. A. Spirin).

[137] "Интегральная геометрия на пространстве $L^{n}$, где $L$ - кольцо матриц", Функи. анализ и его прил., 30:4 (1996), 71-74; англ. пер.: "Integral geometry on the space $L^{n}$, where $L$ is a matrix ring", Funct. Anal. Appl., 30:4 (1996), 277-280.

[138] "Combinatorics of hypergeometric functions associated with positve roots", The Arnold-Gelfand mathematical seminars, Birkhäuser, Boston, MA, 1997, 205-221 (with I. M. Gelfand, A. Postnikov).

[139] “Задача интегральной геометрии на $K^{3}$, связанная с гармоническим анализом на группе $S L(2, K)$, где $K$ - произвольное непрерывное локально компактное поле", Докл. РАН, 352:1 (1997), 15-17 (совм. с И. М. Гельфандом, М. Зыскиным); англ. пер.: "The problem of integral geometry on $K^{3}$ related to Fourier analysis on the group $S L(2, K)$ where $K$ is an arbitrary continuous locally compact field", Dokl. Math., 55:1 (1997), 9-11 (with I. M. Gel'fand, M. Zyskin).

[140] "GG-функции одной переменной", Докл. РАН, 354:2 (1997), 155-158 (совм. с И. М. Гельфандом); англ. пер.: "GG-functions of one variable", Dokl. Math., 55:3 (1997), 344-346 (with I. M. Gel'fand).

[141] " $G G$-функции многих переменных и их связь с общими гипергеометрическими функциями", Докл. РАН, 354:5 (1997), 583-586 (совм. с И. М. Гельфандом); англ. пер.: " $G G$-functions of several variables and their relation to general hypergeometric functions”, Dokl. Math., 55:3 (1997), 406-409 (with I. M. Gel'fand).

$[142]$ " $G G$-функции и их связь с общими гипергеометрическими функциями”, $У M H$, 52:4 (1997), 3-48 (совм. с И. М. Гельфандом); англ. пер.: " $G G$-functions and their relation to general hypergeometric functions", Russian Math. Surveys, 52:4 (1997), 639-684 (with I. M. Gel'fand).

[143] "Разностные и $(r, s)$-аналоги $G G$-функций", Функи. анализ и его прил., 32:1 (1998), 59-62; англ. пер.: "GG-functions and their relation to general hypergeometric functions", Funct. Anal. Appl., 32:1 (1998), 45-48.

[144] “Общие гамма-функции, экспоненты и гипергеометрические функции”, УМН, 53:1 (1998), 3-60 (совм. с И. М. Гельфандом, В. С. Ретахом); англ. пер.: "General gamma functions, exponentials, and hypergeometric functions", Russian Math. Surveys, 53:1 (1998), 1-55 (with I. M. Gel'fand, V. S. Retakh).

[145] "Преобразование Фурье быстро растущих функций и вычисление интегралов, возникающих в теории $G G$-функций”, Докл. РAH, 360:5 (1998), 598-602 (совм. с И. М. Гельфандом); англ. пер.: "Fourier transforms of rapidly increasing functions and the evaluation of integrals in the theory of $G G$-functions", Dokl. Math., 57:3 (1998), 439-442 (with I. M. Gel'fand).

[146] Intermediate inversion formulas in integral geometry, The Erwin Schrödinger International Institute for Mathematical Physics, Vienna, 1998. 
[147] "Специальные функции, связанные с комплексными группами Ли", Докл. РАН, 364:2 (1999), 151-154 (совм. с И. М. Гельфандом); англ. пер.: "Special functions related to complex Lie groups", Dokl. Math., 59:1 (1999), 20-23 (with I. M. Gel'fand).

[148] "Г-полиномы", Вопросы кибернетики. Алгебра. Гипергеометрия. Вероятностъ. Моделирование, М., 1999, 11-30.

[149] "GG functions and their relations to general hypergeometric functions", Lett. Math. Phys., 50:1 (1999), 1-28 (with I. M. Gelfand).

[150] "Полиномы Люка и их связь с общими гипергеометрическими функциями и GG-функциями", Докл. РAH, 372:2 (2000), 151-154 (совм. с И. М. Гельфандом); англ. пер.: "Louck polynomials and their relation to general hypergeometric functions and GG-functions", Dokl. Math., 61:3 (2000), 336-339 (with I. M. Gel'fand).

[151] Избранные задачи интегральной геометрии, Добросвет, М., 2000 (совм. с И. М. Гельфандом, С. Г. Гиндикиным).

[152] "Задача интегральной геометрии, связанная с тройкой грассмановых многообразий", Функи. анализ и его прил., 34:4 (2000), 78-81; англ. пер.: "A problem of integral geometry associated with a triple of Grassmann manifolds", Funct. Anal. Appl., 34:4 (2000), 299-301.

[153] "Задачи интегральной геометрии, связанные с векторными пространствами над некоторыми некоммутативными кольцами", Вопросы кибернетики. Интегральная геометрия. Математические модели. Понимание изображений, Сб. статей, НИИ системных исследований РАН, М., 2001, 102-125.

[154] "Гипергеометрические $\mathscr{N}$-функции", Докл. PAH, 379:1 (2001), 12-15 (совм. с И. М. Гельфандом); англ. пер.: "Hypergeometric $\mathscr{N}$-functions", Dokl. Math., 64:1 (2001), 6-9 (with I. M. Gel'fand).

[155] " $\mathscr{N}$-функции и их связь с решениями общих гипергеометрических систем и $G G$-систем", УMH, 56:4 (2001), 3-34 (совм. с И. М. Гельфандом); англ. пер.: " $\mathscr{N}$-functions and their relation to solutions of general hypergeometric systems and GG-systems", Russian Math. Surveys, 56:4 (2001), 615-647 (with I. M. Gel'fand).

[156] "Гипергеометрические функции над конечными полями", Докл. РАН, 381:6 (2001), 732-737 (совм. с И. М. Гельфандом); англ. пер.: "Hypergeometric functions over finite fields", Dokl. Math., 64:3 (2001), 402-406 (with I. M. Gel'fand).

[157] "Относительно замкнутые операторы, связанные с парой грассманианов", $M a-$ тем. заметки, 71:1 (2002), 61-74; англ. пер.: "Relatively closed operators associated with a pair of Grassmannians", Math. Notes, 71:1-2 (2002), 56-67.

[158] Геометрические и топологические структуры на группоидах, НИИ системных исследований РАН, М., 2002 (совм. с А. В. Когановым).

[159] "Geometric and topological structures related to universal algebras", Russian J. Math. Phys., 10:1 (2003), 57-90 (with A. V. Koganov).

[160] "Геометрические и топологические структуры, связанные с универсальными алгебрами", Докл. РАН, 392:2 (2003), 160-165 (совм. с А. В. Когановым); англ. пер.: "Geometric and topological structures related to universal algebras", Dokl. Math., 68:2 (2003), 182-187 (with A. V. Koganov).

[161] "Problems in integral geometry related to vector spaces over some noncommutative rings", Russian J. Math. Phys., 10:1 (2003), 43-56.

[162] "Бесконечномерные представления алгебры $\mathfrak{g l}(n, \mathbb{C})$ и общие гипергеометрические функции на группе $G L(n, \mathbb{C})$, связанные с комплексными аналогами схем Гельфанда-Цетлина", Докл. РАН, 392:3 (2003), 301-308; англ. пер.: "Infinite-dimensional representations of the algebra $\mathfrak{g l}(n, \mathbb{C})$ and general hypergeometric functions on the group $G L(n, \mathbb{C})$ related to complex analogues of the Gel'fand-Tsetlin schemes", Dokl. Math., 68:2 (2003), 204-210. 
[163] Selected topics in integral geometry, Transl. Math. Monogr., 220, Amer. Math. Soc., Providence, RI, 2003 (with I. M. Gelfand, S. G. Gindikin).

[164] "Infinite-dimensional representations of the Lie algebra $\mathfrak{g l}(n, \mathbb{C})$ related to complex analogs of the Gelfand-Tsetlin patterns and general hypergeometric functions on the Lie group $G L(n, \mathbb{C})$ ", Acta Appl. Math., 81:1 (2004), 93-120.

[165] "Преобразование Радона над локально-компактными абелевыми группами", Докл. РАН, 398:4 (2004), 439-443; англ. пер.: "The Radon transform over locally compact Abelian groups", Doklady Mathematics, 70:2 (2004), 725-729.

[166] "Гипергеометрические функции над произвольным полем", УМН, 59:5 (2004), 29-100 (совм. с И. М. Гельфандом, В. С. Ретахом); англ. пер.: "Hypergeometric functions over an arbitrary field", Russian Math. Surveys, 59:5 (2004), 831-905 (with I. M. Gel'fand, V. S. Retakh).

[167] "Гамма-ряды, общие гипергеометрические функции и $G G$-функции", Maтематические исследования, Сб. трудов, ред. В. Б. Бетелин, НИИ системных исследований РАН, М., 2005, 3-141.

[168] "Геометрические решетки, графы, топологии и метрики, связанные с универсальными алгебрами", Математические исследования, Сб. трудов, ред. В.Б. Бетелин, НИИ системных исследований РАН, М., 2005, 142-196 (совм. с А. В. Когановым).

[169] "General hypergeometric functions of matrices and their connection to representations of linear groups and Lie algebras", Acta Appl. Math., 86:1-2 (2005), 3-19.

[170] "Коммутативная модель представления группы токов $O(n, 1)^{X}$ и обобщенная лебегова мера в пространстве распределений", Функи. анализ и его прил., 39:2 (2005), 1-12 (совм. с А. М. Вершиком); англ. пер.: "A commutative model of a representation of the group $O(n, 1)^{x}$ and a generalized Lebesgue measure in the space of distributions", Funct. Anal. Appl., 39:2 (2005), 81-90 (with A. M. Vershik).

[171] "The basic representation of the current group $O(n, 1)^{X}$ in the $L^{2}$ space over the generalized Lebesgue measure", Indag. Math., 16:3-4 (2005), 499-529 (with A. M. Vershik).

[172] "Структура дополнительных серий и особых представлений групп $O(n, 1)$ и $U(n, 1)$ ”, УМH, 61:5 (2006), 3-88 (совм. с А. М. Вершиком); англ. пер.: "Structure of the complementary series and special representations of the groups $O(n, 1)$ and $U(n, 1)$ ", Russian Math. Surveys, 61:5 (2006), 799-884 (with A. M. Vershik).

[173] "Континуальный аналог схем Гельфанда-Цетлина и реализация основной серии неприводимых унитарных представлений группы $G L(n, \mathbb{C})$ в пространстве функций на многообразии этих схем", Докл. РАН, 412:2 (2007), 154-158; англ. пер.: "A Continuous Analogue of Gelfand-Tsetlin Schemes and a Realization of the Principal Series of Irreducible Unitary Representations of the Group $G L(n, C)$ in the Space of Functions on the Variety of These Schemes", Doklady Mathematics, 75:1 (2007), 31-35.

[174] "Интегральные преобразования на группах $G L(n, \mathbb{R})$ и $G L(n, \mathbb{C})$, связанные с их нильпотентными подгруппами", Вестн. ТамбГУ, 12:2 (2007), 215-221 (совм. с С. В. Кольцовой). 